ACTA UNIVERSITATIS LODZIENSIS

Folia Litteraria Romanica 16, 2021

https://doi.org/10.18778/1505-9065.16.16

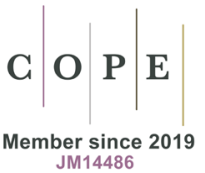

\author{
Agnieszka Woch \\ Université de Łódź \\ (iD) https://orcid.org/0000-0003-0559-9166 \\ agnieszka.woch@uni.lodz.pl
}

\title{
De l'amour pour la patrie dans le discours médiatique polonais
}

\begin{abstract}
RÉSUMÉ
La présente contribution porte sur les stratégies discursives présentes dans le discours des médias numériques qui font appel au sentiment de dévouement envers la patrie. Le discours public polonais reste en étroit rapport avec la mémoire historique de la nation, marquée par les trois partages du pays, par les soulèvements contre les envahisseurs et par les guerres mondiales. Tout cela a certainement influé sur la représentation de l'identité, souvent perçue à travers le prisme de l'amour pour la patrie. Pour cette raison, nous nous proposons d'examiner les stratégies discursives présentes dans le discours de la presse nationale d'actualité afin d'étudier l'emploi des termes " patriote ", " patriotisme » et " patriotique » dans la presse polonaise. Nous nous appuierons sur un corpus d'articles publiés en ligne par des titres représentatifs de la presse numérique dont l'orientation politique est soit de droite, soit de gauche, soit de centre libéral (wSieci, Do Rzeczy, Krytyka Polityczna, Dziennik Trybuna, Kultura Liberalna et Liberté).
\end{abstract}

MOTS-CLÉS - analyse de discours, discours médiatique, patriotisme, stratégies discursives, médias numériques

\section{Love of the Fatherland in the Discourse of Polish Online Media}

\section{SUMMARY}

The present contribution focuses on the discursive strategies used in the Polish online media when they appeal to the feeling of love of the fatherland. The current Polish public discourse on patriotism continues to be marked by historical events such as the three partitions of the country, the uprisings against the invaders, and the two world wars, and the national identity is often perceived through the prism of love for the fatherland. For this reason, in this article the author examines the discursive strategies currently used the Polish national media as well as the meanings that terms such as patriot, patriotism, and patriotic acquire. The corpus-based analysis draws on articles from online newspapers' archives published by representative Polish media from right, left and liberal center political orientations (wSieci, Do Rzeczy, Krytyka Polityczna, Dziennik Trybuna, Kultura Liberalna and Liberté).

KEYWORDS - discourse analysis, media discourse, patriotism, discursive strategies, online media 


\title{
Introduction
}

Dans la présente contribution, nous nous proposons d'étudier l'emploi des termes " patriotisme ", " patriote(s) », et " patriotique(s) » dans le discours médiatique polonais en nous appuyant sur un corpus de 300 articles qui recourent aux termes mentionnés et qui proviennent de la période allant du 1er septembre 2016 au 30 septembre 2019. Parmi les titres de la presse numérique analysée, nous en avons choisi du centre libéral : Kultura Liberalna (KL) et Liberté (L); de gauche : Krytyka Polityczna (KP), Dziennik Trybuna (DT) et de droite : wSieci (S), Do Rzeczy (DRZ). Nous avons retenu pour la constitution du corpus les textes dans lesquels l'emploi d'au moins une des trois occurrences a été attesté.

En se prononçant sur le sentiment de dévouement envers la patrie, Szeligowska constate la présence de « trois grandes traditions patriotiques en Pologne : la démocratie des nobles, le Romantisme et le réalisme politique évoluant vers le nationalisme au début du $\mathrm{XX}^{\mathrm{e}}$ siècle » en observant en plus qu'il :

\begin{abstract}
est intéressant de voir qu'après la fin du communisme, malgré l'effort des intellectuels de forger une nouvelle formule, c'est encore la version romantique qui semble amalgamée avec le concept de patriotisme. Elle souligne le sacrifice et le martyr, s'appuie sur les nombreux échecs polonais dans l'histoire, et se nourrit d'un idéal quasi-mystique de mission. C'est aussi un patriotisme tourné vers le passé, promu dans le cadre de la politique historique des conservateurs, qui voient dans l'histoire un argument à utiliser dans la lutte politique actuelle. Selon certains commentateurs, c'est ce genre de patriotisme qui a surgi instantanément après la catastrophe de l'avion présidentiel (Szeligowska, 2010).
\end{abstract}

Il semblerait que dans l'espace public polonais la description des attitudes, y compris celles patriotiques, soit " déterminée par le passé » (Bralczyk, 2007 : 324). En outre, les acteurs du discours public recourent à la stratégie de l'étiquetage en vue de dévaloriser leur « ennemi ». Ces pratiques discursives sont arbitraires et les étiquettes, parfois injustes, simplifient la réalité et suscitent des émotions négatives chez le destinataire qui n'est pas censé réfléchir à leur véracité. Le sens dénotatif des termes est remplacé par une connotation vague et les symboles historiques sont utilisés en tant qu'armes dans la lutte politique (cf. Bralczyk, 2007 : 301-307). Citons quelques étiquettes stigmatisantes qui circulent depuis un certain temps dans l'espace public, tels « camarades », « bolcheviks », « nomenclature » et surtout « communistes » ou, plus récemment, trois autres termes, à savoir zdrajca 'traître', sprzedawczyk 'celui qui vend sa patrie' et Targowiczanin / Targowica ${ }^{1}$. Les dénominations citées font partie de ce que Bertram qualifie de « culture de mémoire et de stigmatisation » (Bertram, 2016 :

\footnotetext{
${ }^{1}$ Le symbole de la trahison nationale. C'est une référence à la ville de Targowica où dans la nuit du 18 au 19 mai 1792 a été instaurée un complot des nobles polonais, appuyé par la Russie, contre les réformes de la Grande Diète et contre la Constitution du 3 mai 1791.
} 
2, 6-10). Ajoutons que ces termes, tout comme le mot communiste ${ }^{2}$ examiné par Głowiński, n'ont pas « de signification transparente » et peuvent « être utilisés tout à fait librement pour dénommer son adversaire » (Głowiński, 2009 : 216).

\section{La valorisation et la dévalorisation dans le discours médiatique}

On peut se demander si le mot « patriote», se référant à celui ou celle qui « aime sa patrie, se met à son service, prend les armes pour sa défense », tout comme « le patriotisme » désignant un « attachement profond et le dévouement à la patrie, souvent avec volonté de la défendre militairement en cas d'attaque extérieure $»^{3}$ et l'adjectif " patriotique », peuvent devenir également objets de stigmatisation. Pour répondre à cette question, nous avons relevé dans les articles examinés des arguments persuasifs d'ordre affectif, en particulier des figures de style ${ }^{4}$ qui, selon Reboul, s'avèrent non seulement " oratoires en ce qu'elles contribuent à plaire ou à émouvoir, mais [qui] sont [aussi] argumentatives en ce qu'elles expriment un argument en le condensant, en le rendant plus frappant» (Reboul, $2001: 8$ ).

Parmi les figures de style permettant de valoriser ou de dévaloriser en orientant la manière de penser du destinataire, se distinguent dans notre corpus : l'ironie, l'épithètes, les périphrases, les questions rhétoriques et les métaphores. Nous avons également focalisé notre attention sur les mécanismes de valorisation par le lexique. Afin de mettre en relief d'éventuelles différences dans le fonctionnement discursif des termes analysés, nous les avons classés en fonction de leur provenance, notamment ceux de la presse libérale (PL), de la presse de gauche (PdG) et de la presse de droite $(\mathrm{PdD})$.

\section{Le recours à l'ironie}

L'étude du corpus recueilli nous permet de constater que la grande majorité des termes analysés est soit insérée dans un contexte ambigu, soit citée entre des " guillemets ironiques ». Cette ironie qui consiste « à dire le contraire de ce qu'on veut dire, non pour tromper, mais pour railler » (Reboul, 2001 : 239) est employée constamment avec la seule exception d'un groupe d'épithètes valorisantes.

\footnotetext{
2 « Le mot [postcommuniste] même dans la langue de la politique polonaise n'a pas de signification transparente, il peut désigner quelqu'un qui a été communiste et qui est resté fidèle à l'idéologie ancienne, mais il peut également désigner une personne, qui, dans une certaine période, a été liée à l'idée du communisme et ensuite a changé d'opinion en contribuant dans plusieurs cas de façon active à la chute du communisme. En plus, le terme peut être utilisé tout à fait librement pour dénommer son adversaire » (Głowiński, $2009: 216$ ).

${ }^{3}$ Les définitions des termes « patriote » et «patriotisme » proviennent du dictionnaire TLFi [en ligne] et ont été consultées sur le portail du Centre National de Ressources Textuelles et Lexicales CNRTL.

${ }^{4}$ Reboul se prononce ici à propos des métaphores, antithèses et hyperboles.
} 


\subsection{Les épithètes valorisantes}

Dans tous les titres de presse analysés, les épithètes constituent le groupe le plus représentatif. Commençons par le seul cas de l'emploi des épithètes qui ne s'avère pas ironique. La valorisation positive apparaît dans les articles évoquant les personnages historiques ou littéraires, considérés généralement par l'opinion publique comme dévoués à leur patrie :

PL. Ex. 1 : stateczny patriota Skrzetuski 'le patriote posé Skrzetuski' (référence au personnage du roman historique Par le fer et par le feu écrit par Henryk Sienkiewicz) ;

PdG. Ex. 2 : wielcy patrioci 'les grands patriotes' (référence aux hommes politiques Piłsudski, Daszyński, Dmowski, Korfanty, Witos).

Il en va de même pour des personnes et des attitudes considérées par la presse libérale et celle de gauche comme patriotiques. Ce type de patriotisme est qualifié de « quotidien » ou de « constitutionnel » et les patriotes « réels » seraient des citoyens inquiets pour la « démocratie menacée » de leur pays :

PL. Ex. 3 : codzienny konstytucyjny patriotyzm 'le patriotisme constitutionnel quotidien'; PdG. Ex. 4 : rzeczywiści patrioci (...) otwartoglowi, myślący i głęboko poruszeni skala zniszczenia wspólnoty narodowej, zagrożenia rząów prawa i wartości demokratycznych 'les patriotes réels (...) ouverts d'esprit, pensants et profondément troublés par l'ampleur de la destruction de la communauté nationale et par la menace de l'état de droit et des valeurs démocratiques'.

En revanche dans les titres de droite, sont qualifiés de patriotes ou de patriotiques les milieux politiques de la droite polonaise, qui se sont autoproclamés d'ailleurs comme étant «le camp patriotique», et en plus toutes les actions et les événements organisés par les autorités, y compris un pique-nique ou un concert hip-hop :

PdD. Ex. 5 : polscy patrioci 'les patriotes polonais' (le texte mentionne entre autres l'ex ministre de la Défense Antoni Macierewicz et le journaliste de la télévision publique Michał Rachoń) ;

PdD. Ex. 6: wybitny patriota 'un patriote éminent' (référence à l'ex ministre de l'Environnement, des Ressources naturelles et des Forêts Jan Szyszko);

PdD. Ex. 7 : obóz patriotyczny 'le camp patriotique';

PdD. Ex. 8 : piknik patriotyczny 'un pique-nique patriotique';

PdD. Ex. 9 : patriotyczny hip-hop 'un hip-hop patriotique'.

\subsection{Les épithètes dévalorisantes}

Les épithètes de notre corpus apparaissent à haute fréquence dans un contexte propice à l'ironie en remplissant une fonction dévalorisante. C'est en particulier le cas de la presse libérale et de celle de gauche qui critiquent le modèle de patriotisme 
promu par la droite, en qualifiant ses adeptes de patriotes « trop zélés » (ex. 12), « maudits $»^{5}$ (ex. 14), « frimeurs » (ex. 17) ou « nationalistes » (ex. 19) :

PL. Ex. 10 : goracy patrioci 'patriotes ardents';

PL. Ex. 11 : , zarliwy” patriota 'patriote fervent';

PL. Ex. 12 : nadgorliwi ,patrioci” " " patriotes » trop zélés';

PL. Ex. 13 : genetyczny patriota 'patriote génétique';

PL. Ex. 14 : wyklęty patriota 'patriote maudit';

PL. Ex. 15 : konserwatywno-socjalistyczni patrioci 'patriotes conservateurs-socialistes';

PL. Ex. 16 : zawodowi patrioci 'patriotes de profession';

PL. Ex. 17 : patrioci na pokaz 'patriotes frimeurs';

PL. Ex. 18 : lepszy patriota 'meilleur patriote';

PL. Ex. 19 : patriota nacjonalistyczny 'patriote nationaliste';

PL. Ex. 20 : samozwańczy patriota 'patriote autoproclamé'.

La presse libérale reproche en plus à la droite le fait de monopoliser le patriotisme en le traitant de « gouvernemental », « radical » ou « de droite » :

PL. Ex. 21 : patriotyzm rządowy 'patriotisme gouvernemental';

PL. Ex. 22 : radykalny patriotyzm 'patriotisme radical';

PL. Ex. 23 : prawicowy patriotyzm 'patriotisme de droite'.

Quant à la presse de gauche, elle insiste d'une part sur les sèmes de l'hypocrisie (ex. 26, « fausseté patriotique ») et de l'autre, elle dessine la silhouette d'un patriote « typique » (ex. 24 et 25). Elle critique en outre des objets dits « patriotiques » commercialisés dans les magasins qui se désignent en tant que tels. Il s'agit entre autres de lingerie ou bien de battes de baseball aves des symboles nationaux (ex. 27) :

PdG. Ex. 24 : sztandarowy patriota 'patriote phare';

PdG. Ex. 25 : typowy polski patriota 'patriote polonais typique';

PdG. Ex. 26 : patriotyczne zakłamanie 'fausseté patriotique';

PdG. Ex. 27 : patriotyczne kije bejsbolowe 'battes de baseball patriotiques'.

Dans plusieurs textes, surtout publiés dans les titres de gauche, la valeur réelle des épithètes telles que « bon » ou « incorruptible » est détournée par le contexte contradictoire adjacent. Illustrons ce cas par les exemples 28 et 29 :

PdG. Ex. 28 : Zla fryzura i postawa to wystarczajace powody, żeby zaznać śmierci ze strony dobrego patrioty (KP, 1.10.2016) 'Une mauvaise coiffure et une mauvaise attitude sont des raisons suffisantes pour mourir de la main d'un bon patriote';

${ }^{5}$ Référence aux mouvements clandestins et anticommunistes de la résistance polonaise, souvent évoqués dans le cadre de « la politique historique» de la droite et qui font cependant l'objet de controverses, étant donné qu'ils comprenaient non seulement de grands résistants mais aussi des militaires commettant des actes de «banditisme » et qu'ils « avançaient des programmes ouvertement antisémites » (cf. Janicka, $2017: 17)$. 
PdG. Ex. 29 : Ale ponowne rządy prezesa miaty oznaczać również koniec afer winstytucjach publicznych i niekompetentnego zarządzania zasobami państwowymi. Platfomerskich „kolesi” zastapić mieli nieprzekupni patrioci z twardymi zasadami etycznymi (TD, 28.03.2019) 'Mais la nouvelle prise du pouvoir par le président [Kaczyński] visait également à mettre fin aux scandales dans les institutions publiques et à la gestion incompétente des ressources de l'État. Les « copains » de la Plateforme [Civique] devaient être remplacés par des patriotes incorruptibles aux principes éthiques infrangibles'.

Il en va de même pour les deux exemples qui suivent, ironisant sur le patriotisme des « supporteurs fascisants polonais » qui bloquent une marche des fiertés dans la ville de Białystok (ex. 30) et participent à la marche du 11 novembre, organisée pour la Fête de l'Indépendance de la Pologne (ex. 31) :

PdG. Ex. 30 : Polscy, faszyzujący kibole błogosławieni przez księży katolickich i polityków PiS postanowili dać pokazowa lekcję swego patriotyzmu. Zatrzymać „pochód ideologii LGBT", czyli biatostocka Paradę Równości. Parady nie zatrzymali, ale (...) poczęli robić to co potrafia. Czyli grupowo bić kobiety, młodszych i słabszych chłopaków. I rabować im cenne przedmioty. Widać zrabowane telefony komórkowe ci nacjonalistyczni patrioci, mieniacy się spadkobiercami „Żotnierzy Wyklętych”, potraktowali jak wojenne trofea (TD, 25.07.2019) 'Les supporters fascisants polonais, bénis par les prêtres catholiques et par les politiciens du parti PiS, ont décidé de démontrer leur patriotisme en bloquant « le défilé de l'idéologie LGBT », c'est-à-dire la marche des fiertés de Białystok. Ils n'ont pas réussi, alors ils ont commencé à faire ce qu'ils savaient faire le mieux, à savoir : battre des femmes et des garçons plus jeunes et plus faibles et leur voler des objets de valeur. Il paraît que ces patriotes nationalistes, se prenant pour les héritiers des « soldats maudits » ont considéré les téléphones portables volés comme des trophées de guerre';

PdG. Ex. 31 : Jak co roku ogromna większość Polaków zbierze się nie po to, aby wspólnie świętować, ale po to, żeby, niekoniecznie już wspólnie, poogladać, ile polscy patrioci wyrwa drzewek, ile spala aut, ile kamieni wyladuje na głowie policjantów i ile neonazistowskich symboli znajdzie się pośród dzieci, które patriotyczni troskliwi rodzice przyprowadza, w (...) tlum ludzi w kominiarkach (KP, 12.10.2018) 'Comme chaque année, une grande majorité des Polonais se rassembleront non pas pour célébrer ensemble, mais pour regarder, pas nécessairement ensemble, combien de petits arbres les patriotes polonais vont arracher, combien de voitures vont brûler, combien de pierres vont tomber sur les têtes des policiers et combien de symboles néo-nazis seront retrouvés parmi les enfants que leurs parents patriotiques et bienveillants amèneront dans la foule (...) cagoulée'.

Le sentiment de l'amour pour la patrie, qui reste en opposition avec les actions de vandalisme commis par les « patriotes » évoqués dans les textes (comme le vol des objets, l'arrachement des arbres ou le lancement de pierres), dévoile l'intention de « l'énonciateur ironiste » qui transfère d'ailleurs au destinataire « la responsabilité de dégager la portée argumentative » de son énoncé (Bonhomme, 2014 : 185).

En dépit de ce qui précède, nous notons à plusieurs reprises l'emploi des guillemets ironiques censés faciliter la lecture d'un destinataire non initié. Reprenons les exemples cités auparavant : ,Źarliwy” patriota 'patriote « fervent »' (ex. 11), nadgorliwi ,patrioci” “" patriotes » trop zélés' (ex. 12). 
Une fois de plus, dans les titres de droite, nous relevons des guillemets ironiques (ex. 32) et des épithètes dévalorisant les adversaires politiques défavorables aux autorités (ex. 33) :

PdD. Ex. 32 : „rozsądni” patrioci 'patriotes « raisonnables »';

PdD. Ex. 33 : mialki patriotyzm Komorowskiego 'patriotisme peu profond de Komorowski' (référence à l'ex président de la République Polonaise, Bronisław Komorowski).

\subsection{L'emploi ironique de la périphrase, question rhétorique et métaphore}

Parmi d'autres figures de style relevées autour des termes analysés se distinguent les périphrases, les questions rhétoriques et les métaphores portant un jugement de valeur sur le type de patriotisme professé par les patriotes évoqués dans les articles du corpus.

Citons parmi les exemples les plus significatifs de périphrase, deux expressions imagées dont la première fait référence à la strophe écrite en 1774 par Ignacy Krasicki, principal poète des Lumières polonaises, dans laquelle il désigne le patriotisme en tant que « saint amour pour la patrie bien-aimée ». Or dans le contexte contradictoire adjacent, qui informe le lecteur de la possibilité de télécharger sur son portable « une appli » qui vérifie l'origine polonaise des produits achetés, cette expression recherchée acquiert une valeur railleuse :

PL. Ex. 34 : A teraz mogę ściagnąć sobie na telefon apkę, dzięki której będę mogła zweryfikować polskość wszystkich produktów, by nawet podczas banalnych zakupów dawać wyraz ,"świętej milości kochanej ojczyzny” (KL, 5.12.2017) 'Et maintenant, je peux télécharger une appli sur mon téléphone, grâce à laquelle je pourrai vérifier le caractère polonais de tous les produits de sorte que je puisse, même pendant un acte d'achat banal, témoigner mon « saint amour pour la patrie bien-aimée »'.

La seconde périphrase décrit les " patriotes » comme de « fervents admirateurs polonais de leur propre patrie ». L'expression reste en opposition avec la brutalité des actes xénophobes qu'ils avaient commis :

PdG. Ex. 35 : KSENOFOBIA. Kolejna ofiara gorliwych polskich milośników wlasnej ojczyzny padt 35-letni obywatel Ukrainy Andrej Prygon. Doznat ciężkich obrażeń, poturbowany zostat również jego kolega (DT, 10.02.2017) 'XENOPHOBIE. Le citoyen ukrainien Andrej Prygon, âgé de 35 ans, est une autre victime des fervents admirateurs polonais de leur propre patrie. Il a subi de lourdes blessures, son collègue a également été blessé'.

Pour ce qui est des questions rhétoriques, elles acquièrent également un caractère persifleur dans leur contexte immédiat. Celle se référant à l'impolitesse des supporters « patriotiques » en constitue un bon exemple:

PdG. Ex. 36 : Czy patrioci zawsze musza być tacy niemili? (KP, 1.10.2016) 'Les patriotes doivent-ils toujours être aussi impolis ?' 
Une fois de plus, la question apparemment innocente, posée au début de l'exemple 37 : « Qu'est-ce qui est patriotique aujourd'hui ? », est suivie par une série d'interrogations ironiques concernant entre autres l'achat de la crème fraîche produite en Pologne et des tatouages avec des attributs militaires :

PL. Ex. 37 : Co jest dziś patriotyczne? Stowo „patriotyzm” odmienia się przez przypadki od rana do wieczora. Jedni drugim go odmawiaja lub odwrotnie - podziwiaja jego wytwory. Czy kupowanie polskiej śmietany jest patriotyczne? Czy naklejanie na zderzak symbolu Polski Walczacej mieści się w katalogu patriotycznych zachowań? Czy odpowiednio wyrażę swój patriotyzm, jeśli wytatuuję sobie na łydce Małego Powstańca albo szarżę husarii na plecach? (KL, 5.12.2017) 'Qu'est-ce qui est patriotique aujourd'hui ? Le mot «patriotisme » est décliné à tous les cas du matin au soir. Les uns le refusent aux autres ou - tout au contraire - ils admirent ses manifestations. La crème fraîche polonaise est-elle patriotique ? Le fait de placer le symbole de la « Pologne combattante » sur son pare-chocs s'inscrit-il dans le catalogue des comportements patriotiques? Vais-je exprimer correctement mon patriotisme si je me fais tatouer le Petit Insurgé sur mon mollet ou une charge des hussards sur mon dos ?'.

L'exemple le plus frappant de métaphore dans notre corpus est l'image classique de la patrie-mère. Quant au patriote, il est représenté en un enfant allaité. Notons de nouveau le côté ironique de la métaphore : le journaliste plaisante sur le caractère asexué de la mère-patrie en faisant référence à l'attachement (au moins affiché) des " patriotes polonais » aux enseignements de l'église catholique en matière de vie sexuelle :

PL. Ex. 38 : A wiadomo przecież nie od dziś, że jedyne piersi, jakie polscy patrioci ssa, to te Matki Ojczyzny. Oczywiście aseksualne i bezsutkowe (L, 5.08.2017) 'Et on sait depuis longtemps que les seuls seins que les patriotes polonais sucent sont ceux de la mère patrie, bien sûr, asexués et sans tétons'.

\section{La dévalorisation par le lexique}

Pour ce qui est du mécanisme de dévalorisation par le lexique, outre l'utilisation à haute fréquence d'adjectifs (p.ex. frimeur, nationaliste, radical) et plus rarement de substantifs (fausseté), de verbes (battre, blesser) et d'expressions verbales (lancer des pierres) déjà examinés ci-dessus, nous avons relevé deux autres phénomènes linguistiques intéressants.

Il s'agit d'abord du préfixe anti- qui apparaît surtout dans le discours de la presse de droite analysée et qui se réfère aux ennemis politiques des autorités au pouvoir, qualifiés d' " antipatriotiques » :

PdD. Ex. 39 : Patryk Jaki, który określit Platformę Obywatelska jako partię ,, antypatriotyczną" 'Patryk Jaki a qualifié le parti Plateforme Civique d'« antipatriotique »' (DRZ, 25.09.2019);

PdD. Ex. 40 : antypatriotyczna myślozbrodnia 'une *pensée-crime antipatriotique'(DRZ, 26.02.2017) 
En revanche dans le titre d'un article de la Liberté, nous avons relevé un exemple frappant de mot-valise, composé du mot péjoratif kibol 'supporter ultra' et du terme patriotyzm 'patriotisme', à savoir : PL. Ex. 41 : Kibolopatriotyzm (L, 5.12.2017) se référant au type de dévouement pour la patrie, professé par les ultras.

\section{Conclusions}

Le discours public polonais reste en étroit rapport avec la mémoire historique de la nation, marquée par les trois partages du pays, par les soulèvements contre les envahisseurs et par les guerres mondiales. Tout cela influe sur la représentation de l'identité collective, souvent perçue à travers le prisme de l'amour pour la patrie. Les acteurs du débat publique polonais font un appel constant au concept de patriotisme et d'antipatriotisme.

Szeligowska, tout en s'appuyant sur des articles publiés par Mariusz Janicki et Wiesław Władyka ${ }^{6}$, constate qu'après la catastrophe de l'avion présidentiel de 2010, « les fervents promoteurs » de l'amour romantique pour la patrie « ne tenaient pas à convaincre les autres de leur vision du patriotisme, mais l'ont utilisé en tant que mécanisme de différenciation entre les bons et les mauvais patriotes, les "vrais" et les mauvais Polonais » (Szeligowska, 2018). Notre analyse démontre en effet qu'il existe une ligne de division entre deux types de citoyens et deux types de patriotisme qui est d'ailleurs mise en relief par tous les titres des médias numériques examinés. Ainsi, la presse de centre libéral et celle de gauche s'unissent pour refuser aux organes de droite le monopole du terme " patriote » alors que la presse de droite met en question l'amour pour la patrie des adversaires du gouvernement conservateur, adversaires dont le patriotisme serait « tiède ", « peu profond» ou bien « libéral ».

La valorisation positive apparait dans tous les articles concernant les personnages historiques dont le patriotisme ne constitue pas l'objet de controverses majeures pour l'opinion publique. Néanmoins, le partisan des autorités au pouvoir est systématiquement connoté positivement dans la presse de droite tandis qu'il est dévalorisé dans le discours de la presse libérale et celle de gauche qui emploient à haute fréquence le terme « patriote » pour désigner les nationalistes et les ultras. Enfin, la presse de droite qualifie d'une manière explicite les adversaires de son milieu politique d' " antipatriotes » commettant des actes « anti-polonais ».

La valorisation se manifeste dans le corpus par l'emploi de figures de style, mais surtout d' épithètes et de l'ironie. Dans les discours analysés, le mot « patriote » devient ainsi une sorte d'étiquette, à la fois noble et stigmatisante, en fonction de l'orientation politique de la presse examinée. Tous les énonciateurs

\footnotetext{
${ }^{6}$ Mariusz Janicki et Wiesław Władyka, « To ta sama IV RP », Polityka du 8 mai 2010 et « Pokolenie PP », Polityka du 1 mai 2010.
} 
recourent à la fonction expressive, en employant des arguments persuasifs d'ordre émotionnel en vue d'influencer la manière de penser du destinataire.

Terminons nos réflexions sur l'amour pour son propre pays en citant les paroles prononcées par une grande figure des lettres polonaises, Wiesław Myśliwski. Cet écrivain et dramaturge, lauréat de nombreux prix littéraires et, vu son âge (il est né en 1932), témoin de plusieurs impasses historiques de la Pologne, a avoué : « Je n'aime pas le mot "patriotisme", il est déjà invraisemblablement usé. C'est le mot le plus malade de la langue polonaise $»^{7}$.

\section{Bibliographie}

BERTRAM, Łukasz (2016), « Targowiczanie z UB, komuchy na pasku hitlerowców. Radykalizacje historyczne w polskiej debacie publicznej », Kultura Liberalna. (https://obserwatorium. kulturaliberalna.pl/raport/targowiczanie-z-ub-komuchy-na-pasku-hitlerowcow/, consulté le 11 février 2020)

BONHOMME, Marc (2014), Pragmatique des figures du discours, Paris, Honoré Champion

BRALCZYK, Jerzy (2007), O języku propagandy i polityki, Varsovie, Trio

GŁOWIŃSKI, Michał (2009), Nowomowa i ciagi dalsze. Szkice dawne i nowe, Cracovie, Universitas JANICKA, Elżbieta (2017), L'innocence retrouvée. L'affaire Gross comme produit de la politique historique de l'État polonais depuis 2004. Paris, L'École des Hautes Études en Sciences Sociales. (https://www.ehess.fr/sites/default/files/pagedebase/fichiers/janicka_gross_final-1. pdf, consulté le 10 février 2020)

JANICKI, Mariusz, WŁADYKA, Wiesław (2010), « Pokolenie PP », Polityka, 1.05.2010

JANICKI, Mariusz, WŁADYKA, Wiesław (2010), « To ta sama IV RP », Polityka, 8.05.2010

REBOUL, Olivier (2001), Introduction à la rhétorique, Paris, PUF

SZELIGOWSKA, Dorota (2010), «Lech Kaczyński post-mortem : "Un mauvais Président, mais un bon patriote" ?» (http://www.nouvelle-europe.eu/lech-kaczynski-post-mortem-un-mauvaispresident-mais-un-bon-patriote, consulté le 8 février 2020)

Agnieszka Woch - professeure des universités à l'Institut d'Études Romanes de l'Université de Łódź (Pologne), HDR en linguistique, docteure ès sciences humaines de l'Université de Łódź et de l'Université Paris Descartes (spécialité : linguistique). Auteure et co-rédactrice de six monographies et d'une quarantaine d'articles. Ses principaux domaines d'intérêt scientifique sont l'analyse du discours, la pragmatique et la sociolinguistique. Ses recherches actuelles portent sur le discours médiatique et politique.

7 « Nie lubię słowa « patriotyzm», jest już nieprawdopodobnie zużyte. To jest najbardziej chore słowo w polszczyźnie ». L'interview avec Myśliwski a été publié le 16 octobre 2018 dans le magazine Książki 'Livres' de Gazeta Wyborcza. 